\title{
PID DC motor drive with gain scheduling
}

\author{
Wasif Abdel Aziz Saluos ${ }^{1}$, Mohammad Abdelkarim Alia ${ }^{2}$ \\ ${ }^{1}$ Faculty of Engineering Technology, Zarqa University, zarqa Jordan \\ ${ }^{2}$ This research is Supported by Zarqa University, zarqa-Jordan \\ Email address: \\ Saluos2000@yahoo.com (W. A. Saluos), makalalia2000@yahoo.com (M. A. Alia)
}

\section{To cite this article:}

Wasif Abdel Aziz Saluos, Mohammad Abdelkarim Alia. PID DC Motor Drive with Gain Scheduling. American Journal of Software Engineering and Applications. Vol. 3, No. 6, 2014, pp. 102-105. doi: 10.11648/j.ajsea.20140306.16

\begin{abstract}
In this work software-based PID controller with gain scheduling is implemented to drive a DC motor. LabVIEW PID controller tool with its associated gain scheduling VI was used. Motor start up interval was experimentally analyzed and divided into three regions with three related PID gains sets. Gain scheduling selection criterion was based on the dynamic error absolute value, and it was realized by using case structures. Experiments show that speed overshoot was eliminated and drive system response became faster. Generally it is possible to auto tune the PID controller to achieve a response with the required static or dynamic specifications.
\end{abstract}

Keywords: PID Control, Gain Scheduling, PWM, Adaptive Controller

\section{Introduction}

Although PID controllers are by far the most widely used controllers in industry, they are not always well suited for every process control system. One important drawback of PID control is the frustration often involved in reaching the correct tuning parameters for a given operation. Because of that academics have been working on countless alternatives.

As an example of these attempets is our work (2), where aself-tuning software based PID controller was designed . This work may be considered as continuation of the previous one aiming at exploring the possibility of realizing an adaptive controller with gain scheduling.

The advent of microprocessors and advanced computing platforms has catalyzed the shift to adaptive model-free PID controllers for its ability to improve the performance of mechanical and electromechanical systems ( 1, 2 ).Recently, gain scheduling technique is benefiting from the advances in PC computing dedicated software and is used generally in non-linear control systems successfully. One of the inherent characteristics of DC motor drives is the undesirable speed overshooting when it is required to design a dynamic-mode high speed motor drive systems. In order to get a high speed motor drive with minimum overshoot, gain scheduling is suggested to be used in this work. In this case one can tune the PID controller for different sets of controller parameters . Depending on the value of the dynamic error during the transient period, and by using an if - then statement ( case structure ) the corresponding set of PID parameters shall be called by the controller in order to achieve the required performance. In order to realize this idea LabVIEW gainscheduled and PID software are utilized. Experimental results show that system performance has improved compared with tuning PID controller only with one set of parameters.

\section{Description of the Motor Drive System}

The block diagram of the drive system is shown in figure NO (1). The main hardware components are a DC motor , tacho- generator, a PC , NI DAQ board and power interface circuit which includes a MOSFET- static power switch. The controller is software- based using LabVIEW PID tool with its associated gain scheduler. The output

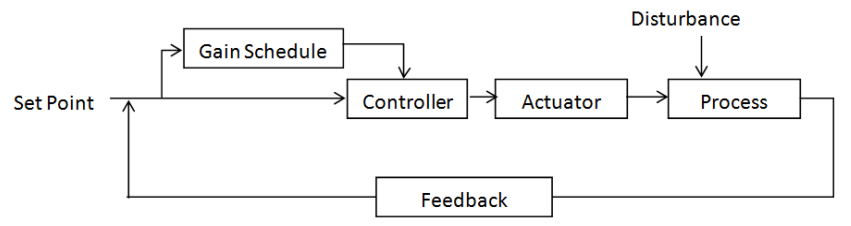

Figure NO (1). Block diagram of the control system

Of the PID is the driving signal of a pulse width modulator( PWM )which is generated internally within LabVIEW. The PWM output is fed to the power interfacing circuit. As the PID output signal changes the PWM duty 
cycle changes proportionally regulating by that the average voltage which feeds the motor armature,. Consequently the motor speed is controlled. As the block diagram shows, the gain scheduler is connected in a feed forward channel and functions depending on the value of the dynamic error. As seen, the general setup of the drive system is quazi -invariant with a feed forward and feedback configurations. Such a drive system may be viewed as a feedback system in which the feedback gains are adjusted by using feed-forward compensation.

Although the feedback configuration helps to compensate for variation of system parameters and system components non-linearity, the inclusion of an adaptive feed forward channel will also reduce the effects of parameter variation which makes the control system more robust.

\section{Software Components}

The block diagram of the control system is shown in figure $\mathrm{NO}(2)$. It includes the following components. The DAQ assistant express VI, PID controller ,numeric functions, case structures, mean value VI, comparison functions, waveform chart and simulate signal VI.

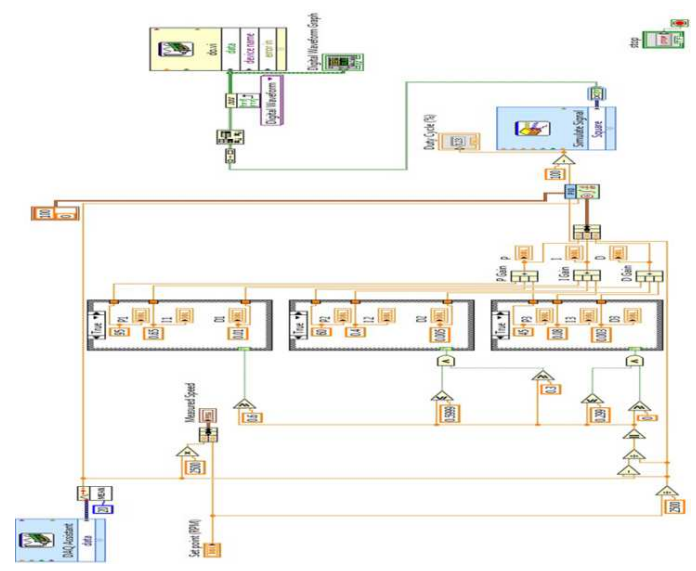

Figure NO (2). The block diagram of the control system

The front panel of the control system is given in figure NO (3). It includes the controls and indicators .

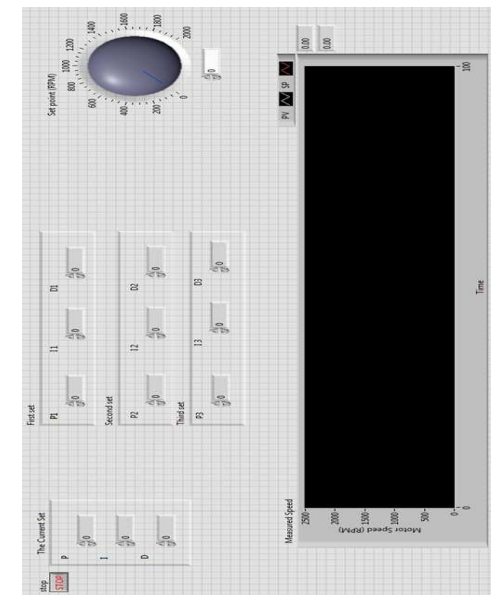

Figure NO (4). Front panel widow of the control system
The DAQ assistant is in charge of reading the voltage from the tacho-generator output. Morover the mean component takes the average of the read voltage every 20 samples.

Some numeric and logic functions(comparison) are involved in order to convert the feedback voltage into a speed measured in rotations per minuite( RPMs ).

This is shown in figure $\mathrm{NO}(3)$.

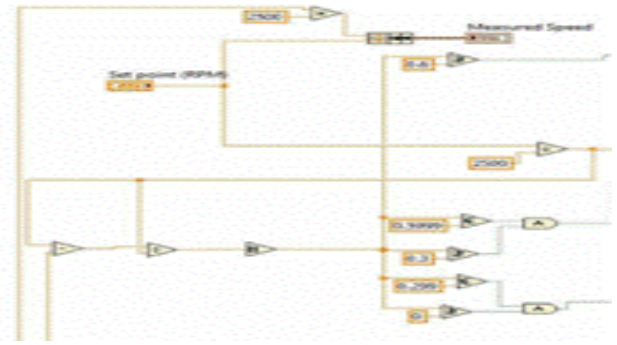

Figure NO (3) Mathematic \& Comparisons Operations

For the gain scheduling selector to function, speed error is found as the difference between the set point and the process variable i.e. the instantaneous speed.

$\varepsilon=S P-P V$. The absolute value of the error is divided by the set point to get a ratio

$$
x=\frac{S P-P V}{S P}=\frac{\varepsilon}{S P} ; 0 \leq x \leq 1
$$

For gain scheduling we used three sets of PID parameters which correspond to three sets of ratio $\mathrm{x}$ such that :

- For the first PID parameters set $0.6 \leq \mathrm{x} \leq 1$

- For the second PID parameters set $0.3 \leq \mathrm{x} \leq 0.599$

- For the third PID parameters set $0 \leq \mathrm{x} \leq 0.295$

Concerning the case structure, the selector of the desired set is connected to case structure. The case structure has two states ( True, False) states. For the False state the value (0) is inserted in it. For the True state we entered the values of ( $\mathrm{x}$ ) sets. Thus we have one case structure for each set as shown in figure NO (5).

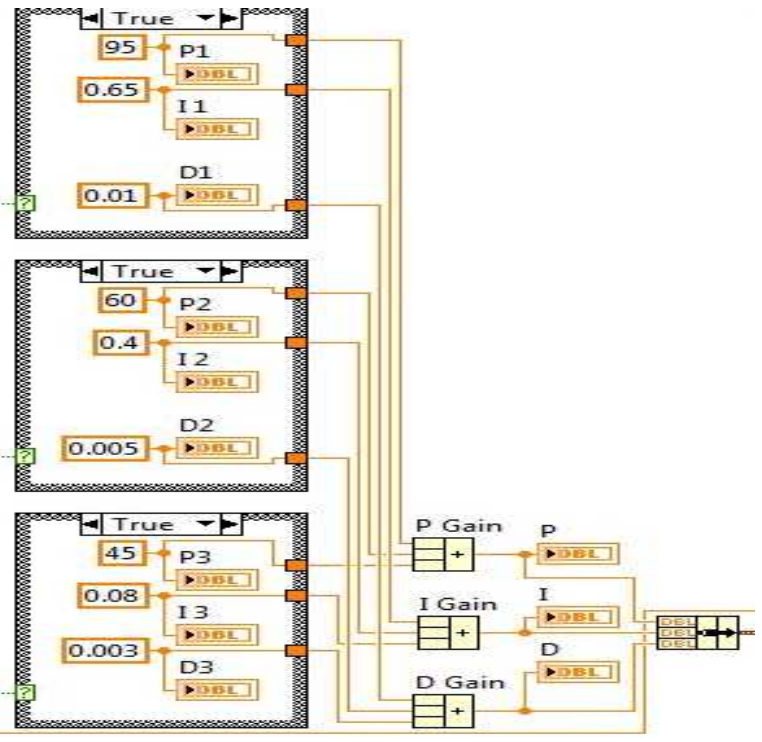

Figure NO (5). Details of the case structure connections 
The PID virtual instrument has three inputs :

- The set point, which is connected to a dial control that is located at the front panel.

- Process variable, which is represented by the data coming from the DAQ assistant.

- PID parameters( gains ) which are the data coming from the output of bundle component.

The output of the PID VI is utilized in order to control the duty cycle of the PWM signal. The PWM signal is generated internally using Simulate Signal

Express VI .In order to get the control signal out of the DAQ device the DAQ assistant with some conversion signal tool was utilized.

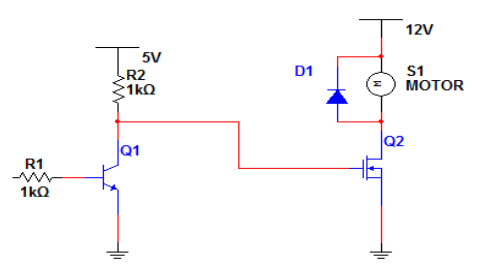

Figure NO (6). Power interface circuit.

Concerning power interfacing, simple power interfacing circuit is given in figure NO (6).

\section{Experimental Results}

Initially the values of the set point and process variable are zero. When the desired speed is set through the dial control in the front panel, the motor starts running and motor speed keeps increasing until it reaches the set point. During the rise time the speed error begins decreasing from the maximum value up to zero.

At the first stage, when the error is maximum( the ratio of the error to the set point is between 0.6 and one, the system will select the first set of gains, which is immediately displayed on the Operating Set Indicator. When the speed reaches the middle of the rise time the ratio $(\mathrm{x})$ will be in the second range and the gain scheduling selector will select the second set of gains. At the final portion of the rise time the third set of PID parameters is selected and the speed creeps closer towards the set point.

For an open loop control circuit Figure NO (7) shows the PWM and steady state speed for a duty cycle of $20 \%$ and a speed of 1209 RPM.

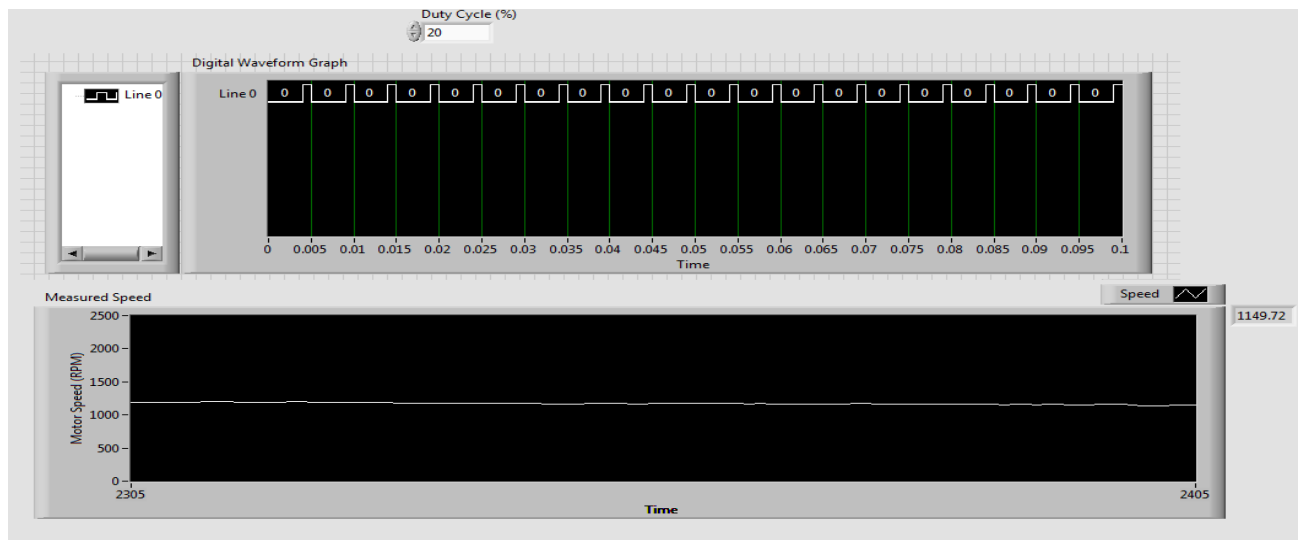

Figure NO (7). PWM signal and motor speed at $20 \%$ duty cycle

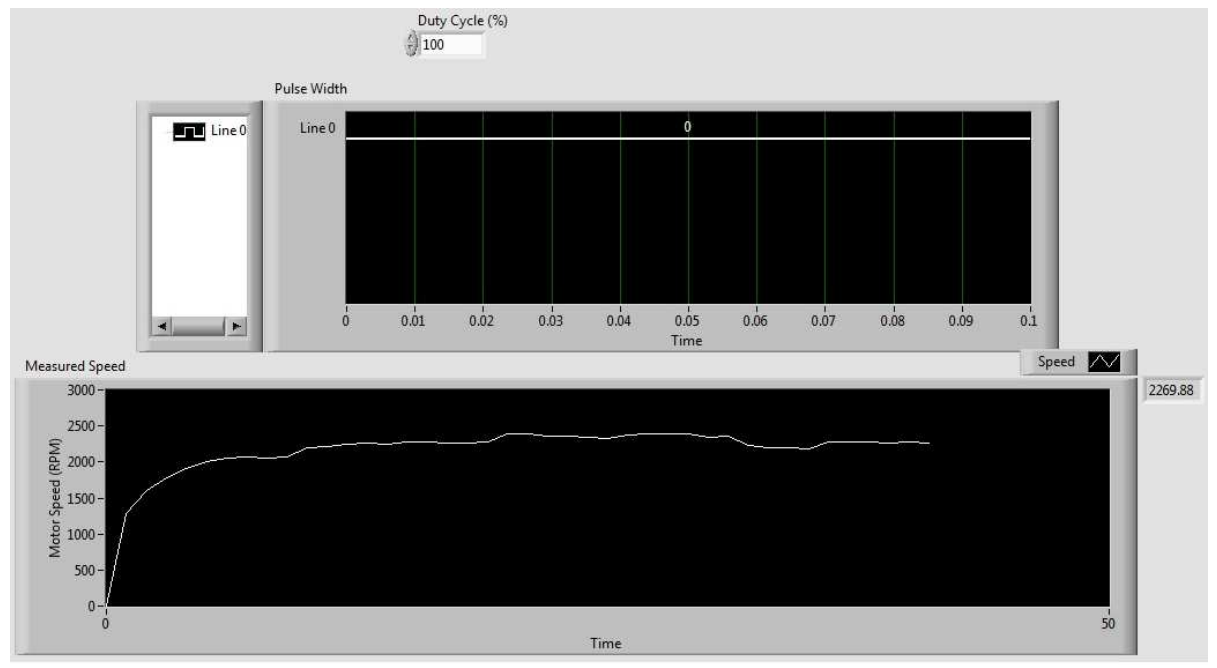

$100 \%$ of duty cycle

Figure NO (8). Showes the PWM signal and motor speed at 100\% duty cycle. 
Figure NO (8) PWM signal and motor strat up speed at $100 \%$ duty cycle

For a closed loop system figure NO (9) shows the values of the gains for the three sets of PID gains.

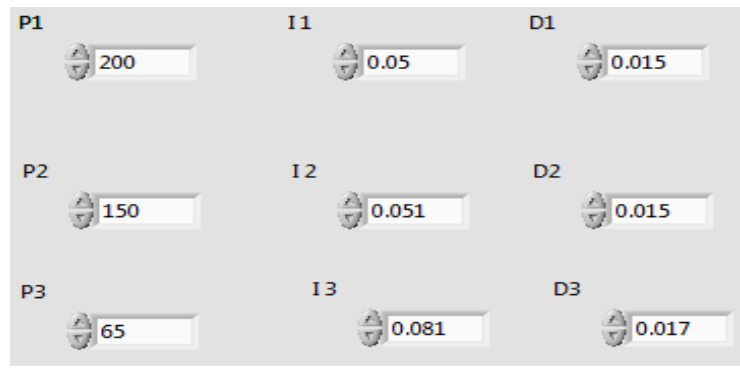

Figure NO (9). Indicator of the values of used PID gains

Figure NO (10 ) shows motor speed at start up and steady state regions for a closed loop system when three PID gain settings are used. The figure shows that the over shoot is not existing.

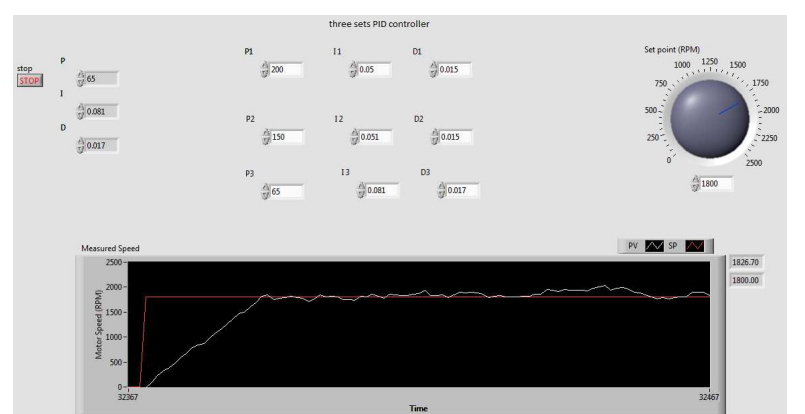

Figure NO (10). Start up speed curve when three PID gain settings are used

In order to compare between gain scheduled and one gain setting PID controller, curve NO (11 ) shows the start up curve for the first set of PID gains only.

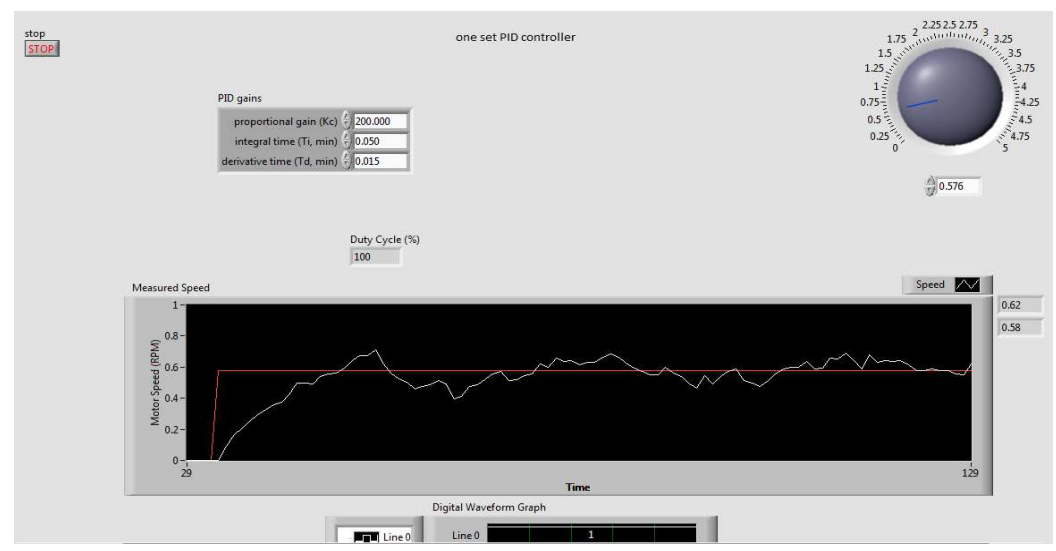

Figure NO (11). Motor start up with the first set of PID gains

\section{Conclusions}

Using a PID controller with gain scheduling improves the performance of the control system. The system becomes faster with minimum overshoot, which means that the system becomes more robust with a higher stability margin.

Using PID gain scheduling is applicable for linear or nonlinear control systems. By dividing the system working interval into a specific number of regions with the appropriate sets of PID gains, controller tuning becomes an automatic process and the iterative tuning procedure is eliminated.

\section{References}

[1] Simulation of auto-tuning PID controller for DC motor using Ziegle-Nichols method. Aina Azliyana bt Mohamd. Thesis of B.SC degree. University Malaysia Pahang. June - 2012
[2] A design of a PID self-tuning controller using LabVIEW. Mohammad A.K.Alia, Tariq M.Younes, Shebel A.Alsabbah. Journal of software engineering and applications. March2011, 4, 161-171

[3] Comparison performance of different PID controllers for DC motor. Abidoun H.Shellal. Diyala Journal of engineering sciences. June -2012, Vol. 5 NO 01 Diyala University.

[4] Study on auto tuning controller for servo system. Nguyen Hoang Giap, Jin-Ho Shin , Won -Ho-Kim. Intelligent control and automation . July 2014, $5,102-110$. Graduate school of Dong-eui University. Busan-Korea

[5] Adaptive PID controller for DC motor speed control. A Taifour Ali, Eisa Bashier M and others International journal of engineering inventions. Sept 2012 , Vol. 1 , issue 5

[6] Refinments of Ziegler - Nichols tuning formula . Hang C-C K.J Astrom and W.K Ho. IEE proc . part D , Vol. 138, NO 2pp, March 1991

[7] PID control system analysis, design and technology.IEEE transactions on control systems technology. Vol. 13, NO 4 July2005. 